\title{
41. Prise en charge de l'insuffisance cardiaque
}

\author{
(C) Springer-Verlag France 2010
}

\section{1}

\author{
Insuffisance cardiaque : bilan à faire et suivi \\ C. Swine \\ UCL/IRSS, Belgique
}

Le syndrome d'insuffisance cardiaque (IC) est fréquent en gériatrie et ses manifestations ne sont pas toujours typiques. Il n'est pas rare que le diagnostic soit posé à l'occasion d'une chute, d'un déclin fonctionnel ou d'un delirium. Le diagnostic de l'IC repose néanmoins principalement sur des critères cliniques (dyspnée, congestion, œdèmes, fatigue) associés à une atteinte cardiaque préexistante (coronaropathie, hypertension, valvulopathie), et/ou de manière aigue (OAP) à la faveur de facteurs précipitants (FA, anémie, AINS, influenza...). L'amélioration clinique et la perte de poids sous diurétiques valideront rétrospectivement le diagnostic. Le dosage du BNP permettra de trancher les cas douteux. La radiographie de thorax ajoute au diagnostic clinique, mais sa spécificité n'est pas excellente. Quant à l'ECG il a une valeur prédictive négative (normal = pas d'IC). Dans le bilan, l'échocardiographie renseigne sur le type d'IC (systolique, diastolique), sur les atteintes structurelles (hypertrophie, hypokinésies, dysfonctions valvulaires) et l'hémodynamiques (pressions, débit, performances diastoliques).

Le suivi de l'IC en gériatrie vise la prévention des complications et des réhospitalisations dont à défaut elle est pourvoyeuse. Le carnet type suit le poids, la prise des médicaments, les symptômes d'alerte et des événements précipitants. Les proches à domicile, l'infirmière et le médecin de famille jouent un rôle important à cet égard. Enfin, le clinicien repérera à temps les cas d'IC terminale, afin de dispenser les soins de confort en contrôlant les symptômes pénibles tout en évitant les examens superflus et les réanimations inutiles.

\author{
41.3 \\ Stratégie thérapeutique pour l'insuffisance \\ cardiaque $\mathrm{C}$ systolique \\ O. Hanon \\ Service de gériatrie, université Paris-5, hôpital Broca, Paris
}

Même si, aucun essai thérapeutique n'a été spécifiquement réalisé chez des insuffisants cardiaques âgés de 80 ans et plus, toutes les études qui ont inclus des malades âgés indiquent un effet bénéfique des traitements par IEC (inhibiteurs de l'enzyme de conversion (IEC) et bêtabloquants $(\beta B)$ lorsque la fonction systolique est altérée (FEVG $<50 \%)$, et ce quel que soit l'âge des malades. Dans la cohorte européenne de l'Euro Heart Failure Survey, la mortalité des 2780 insuffisants cardiaques de 80 ans ou plus (âge moyen : 85 ans) était significativement diminuée sous IEC $(\mathrm{OR}=0,60[0,46-0,80])$ et sous $\beta \mathrm{B}(\mathrm{OR}=0,70[0,49-1,0])$. Dans l'étude SENIORS, menés chez des sujets de plus de 70 ans (âge moyen $=75$ ans) dont $20 \%$ d'octogénaires, le risque de décès ou d'hospitalisations pour causes cardiovasculaires (critère principal) a été significativement réduit de $14 \%$ par un bêtabloquant (le nébivolol) par rapport au placebo $(\mathrm{RR}=0,86[0,74-0,99])$.

Pourtant, les IEC et les $\beta$ B sont insuffisamment prescrits chez les insuffisants cardiaques âgés. En pratique, il existe pourtant peu de contre-indication à la prescription de ces traitements, mais, des précautions particulières concernant la posologie et la surveillance sont nécessaires en raison des comorbidités et des modifications pharmacocinétiques ou pharmacodynamiques liées au vieillissement. En l'absence d'étude spécifique, la posologie optimale correspond à la posologie maximale tolérable, si possible proche de celle atteinte dans les grandes études de mortalité. De façon générale, une surveillance clinique (signes d'insuffisance cardiaque, hypotension orthostatique, fréquence cardiaque) et biologique (ionogramme sanguin, créatinine) doit être proposée régulièrement. 